\title{
ANÁLISIS DEL AUTOCONCEPTO Y REALIZACIÓN PERSONAL: NUEVOS DESAFÍOS EN LA FORMACIÓN INICIAL DEL PROFESORADO
}

\author{
$\mathbf{M}^{\mathrm{a}}$ del Carmen Pegalajar Palomino \\ Universidad de Jaén
}

\begin{abstract}
RESUMEN: La formación inicial del profesorado debe favorecer su desarrollo integral, potenciando la adquisición de contenidos académicos así como la mejora de habilidades emocionales. Este trabajo pretende analizar valores asociados al autoconcepto y la realización personal en estudiantes del Grado de Educación Infantil de la Universidad Católica de Murcia $(\mathrm{N}=270)$. Para ello, se ha utilizado como instrumento de recogida de datos el "Cuestionario de Autoconcepto y Realización" (Domínguez, 2001). Los datos obtenidos revelan resultados positivos al analizar los niveles de autorrealización y crecimiento personal del alumnado del Grado de Educación Infantil; los aspectos mejores valorados por los estudiantes se relacionan con la sociabilidad y comunicación, respeto, colaboración e identificación, afecto, valoración positiva y ayuda. De igual modo, se obtiene puntuaciones muy favorables al cuestionar la disponibilidad del estudiante ante la tarea, motivación y evaluación de logro, planificación, precisión en la acción y evaluación del trabajo. Además, se comprueba reciprocidad entre las distintas dimensiones incluidas en el instrumento de recogida de datos. Tal análisis puede servir de interés para el desarrollo de experiencias de aprendizaje basadas en nuevas técnicas de atención consciente "mindfulness" en el Grado de Educación Infantil, pues favorece la promoción del bienestar emocional en el alumnado.
\end{abstract}

PALABRAS CLAVES: Autoconcepto, realización personal, profesorado, Grado en Educación Infantil, formación.

\section{SELF AND PERSONAL REALIZATION ANALYSIS: NEW CHALLENGES IN INITIAL TEACHER TRAINING}

ABSTRACT: The initial teacher should encourage their development, promoting the acquisition of academic content and improving emotional skills. This paper analyzes values associated with the self-concept and selfrealization in students of Early Childhood Education Degree at the Catholic 
University of Murcia ( $\mathrm{N}=270)$. To this end, it has been used as an instrument of data collection the "Self-concept and realization Questionnaire" (Dominguez, 2001). The data obtained show positive results when analyzing levels of self-realization and personal growth of students in Early Childhood Education Degree; the aspects most highly valued by students relate to the sociability and communication, respect, cooperation and identification, affection, positive assessment and support. Similarly, very favorable scores are obtained to question the availability of the student to the task, motivation and achievement assessment, planning, action and precision in job evaluation. In addition, reciprocity is found between the different dimensions included in the data collection instrument. Such analysis may be of interest for the development of learning experiences based on new techniques of mindfulness in Early Childhood Education Degree, it favors the promotion of emotional well-being in students.

KEYWORDS: Self-concept, self-realization, teacher, Early Childhood Education Degree, training.

Recibido: 16/03/2016

Aceptado: 19/07/2016

Correspondencia: $M^{a}$ del Carmen Pegalajar Palomino, Universidad de Jaén, Facultad de Humanidades y Ciencias de la Educación, Departamento de Pedagogía, Campus "Las Lagunillas", s/n. C.P. 23071 Jaén. Email: mcpegala@ujaen.es.

\section{INTRODUCCIÓN}

El término de Inteligencia Emocional ha sido definido por Mayer, Salovey y Caruso (2000: 398) como "la capacidad para procesar la información emocional con exactitud y eficacia, incluyéndose la capacidad para percibir, asimilar, comprender y regular las emociones". Asimismo, Bisquerra (2000) estructura las competencias emocionales de la siguiente manera:

- Conciencia y control emocional, vinculado al conocimiento de las propias emociones y las de los demás;

- Regulación emocional, centrado en el dominio de la expresión de las emociones y al proporcionar una respuesta adecuada a la situación;

- Autonomía personal, en la que se incluyen la autoestima, automotivación, actitud positiva, responsabilidad, análisis crítico de normas sociales, buscar ayuda y recursos y auto-eficacia emocional;

- Inteligencia interpersonal, relacionada con el dominio de habilidades sociales básicas, el respeto por los demás, comunicación receptiva, comunicación expresiva, compartir emociones, comportamiento pro-social y cooperación y asertividad; 
- Habilidades de vida y bienestar, relacionadas con la identificación de problemas, fijación de objetivos adaptativos, solución de conflictos, negociación, bienestar subjetivo y experiencia óptima.

Las personas emocionalmente competentes pueden reflexionar sobre cómo y cuándo usar, de un modo efectivo, habilidades como la automotivación, control de impulsos, independencia, iniciativa, perseverancia, obtener el máximo rendimiento de sus capacidades, saber traducir el pensamiento en acción, completar tareas, no temer al fracaso, no postergar, aceptar el reproche justo, rehusar la autocompasión, equilibrar el trabajo, aplazar la gratificación, tener autoconfianza y creencia para alcanzar metas, superar las dificultades personales y equilibrar el pensamiento analítico, creativo y práctico (Sternberg, 1997).

El contexto educativo representa un lugar privilegiado para la promoción de estas habilidades y el desarrollo de competencias socioemocionales, pues contribuyen de forma positiva al bienestar personal y social de las personas (Cassullo y García, 2015). Para ello, el docente deberá replantearse sus valores, separándose de los menos convenientes y acercándose a los más positivos y favorables desde el punto de vista académico y social (Martínez, 2015).

De manera más concreta, la inclusión de las competencias emocionales en la formación inicial del profesorado resulta acertada (López y Goñi, 2012), pues implica el desarrollo de las capacidades de conocimiento más allá de lo puramente teórico (Palomares y Garrote, 2009). Se centra en el desarrollo de la conciencia y capacidad para gestionar y controlar sus propias emociones y sentimientos, la motivación para afrontar dicho proceso así como las relaciones interpersonales con los demás (Soriano y Osorio, 2008).

Las competencias más adecuadas para el desempeño de la profesión de maestro de Educación Infantil están relacionadas con el desarrollo para la competencia didáctica, emocional así como aquellas otras vinculadas a la identidad profesional, innovación, investigación y respuesta a la interculturalidad (Domínguez y García, 2012; Medina, Domínguez y Sánchez, 2013). Mieles, Henriquez y Sánchez (2009) entienden que para que el docente de Educación Infantil sepa qué hacer y cómo, qué enseñar y a quiénes así como cuáles son los métodos, técnicas y estrategias más adecuadas, es necesario que pueda identificar sus propias necesidades y tener la capacidad de hacerse cargo de su propia experiencia emocional.

Distintas investigaciones (Extremera y Fernández-Berrocal, 2004; Palomera, Fernández-Berrocal y Brackett, 2008; Poulou, 2005) indican el reconocimiento que Ilevan a cabo los docentes sobre la importancia de la inteligencia emocional para el ejercicio de su profesión. No obstante, la formación del docente hacia el desarrollo de estas capacidades debe tener un componente teórico y otro vivencial (Fernández, Palomero y Teruel, 2009); así pues, y tal y como indica Zabalza (2006), la dimensión personal puede provocar dificultades en la comunicación, en las relaciones interpersonales, etc., afectando de manera directa al tipo de docencia que se lleva a cabo.

El desánimo profesional del docente afecta a las percepciones que éste tiene acerca de su autoconcepto, lo que le provoca inseguridad y falta de autoestima (Hué, 2008). Peñalva, López y Landa (2013) demuestran cómo uno de cada dos alumnos 
de Magisterio presenta un déficit en las competencias emocionales que incluyen habilidades personales e interpersonales, si bien las habilidades en este área son superiores en comparación a la población universitaria en general (Bueno, Teruel y Valero, 2005). Incluso, Pertegal, Castejón y Martínez (2009) muestran cómo los estudiantes no están preparados para una integración laboral exitosa en cuanto a competencias emocionales se refiere; no tienen las habilidades suficientes para trabajar en equipo, manejar con personas, adaptarse a los continuos cambios propuestos o controlar sus emociones, entre otras.

Una mayor competencia emocional por parte del docente implica un menor agotamiento y despersonalización, además de mayores niveles de realización personal (Pena y Extremera, 2012). El docente que se percibe a sí mismo como poseedor de cierto grado de habilidades para regular sus emociones utiliza más estrategias activas para enfrentarse a situaciones estresantes en el entorno académico (Mearns y Cain, 2005). Además, según Bisquerra (2007), cuando los profesores han adquirido competencias emocionales están en mejores condiciones para relacionarse con el alumnado, con el resto del profesorado y con las familias. Además, puede contribuir al desarrollo de las competencias emocionales del alumnado la utilización de técnicas activas basadas en la reflexión, introspección, juegos, relajación, concentración, respiración y desarrollo de actividades prácticas de interacción social (Cassullo y García, 2015).

Por ello, se hace necesario, el diseño de programas de promoción del bienestar psicológico en el alumnado como medio, a la vez, de mejorar la calidad de la enseñanza universitaria (Salami, 2010). Las nuevas técnicas de atención consciente "mindfulness" permiten alcanzar un estado de relajación corporal, en el que la mente está tranquila y concentrada y se pueden percibir las sensaciones del momento presente (Thera, 2008). Dichas técnicas consiguen integrarse en el contexto educativo a partir de tres modalidades, tal y como establecen Meiklejohn et al. (2012):

- Indirectamente, pues el profesor practica de forma personal mindfulness y representa sus actitudes y conductas en el contexto educativo.

- Directivamente, a través de la enseñanza al alumnado de ejercicios y habilidades basados en la meditación.

- De modo combinado, pues profesores y alumnado practican mindfulness.

Distintos estudios científicos revisados sobre la práctica "mindfulness" ofrecen un sólido conjunto de resultados prometedores en el bienestar emocional, la capacidad y potencial de aprendizaje y en la salud física y mental de quienes lo practican (Burnett, 2010). Franco, De la Fuente y Salvador (2011) han reflejado que el programa es válido y adecuado para promover el crecimiento y autorrealización personal de aquellos que lo practican. Por su parte, Langer, Ulloa, Cangas, Rojas y Krause (2015) muestran cómo el mindfulness, al ser aplicado como estrategia de prevención en contextos educativos para jóvenes, produce cambios significativos ante variables psicosociales, al fomentar el aumento de las habilidades sociales. Además, Shapiro, Browm y Astin (2008) aluden a los efectos positivos de la meditación para el rendimiento cognitivo y académico del alumno, la salud mental, el bienestar psicológico y el desarrollo integral u holístico de la persona. 
Este trabajo pretende analizar el autoconcepto y la realización personal en estudiantes del Grado de Educación Infantil de la Universidad Católica de Murcia. Se trata de una investigación basada en una metodología descriptiva-correlacional la cual trata de alcanzar varios objetivos específicos tales como:

- Examinar las percepciones sobre autorrealización y crecimiento personal en la formación inicial del profesorado.

- Conocer el grado de relación entre las apreciaciones del alumnado hacia distintos factores sobre autoconcepto y realización personal, siendo éstos: Amistad y Amor, Actitud ante la Tarea, Autoconcepto, Fuerza del Yo, Proyectos y Preocupación Social.

- Establecer propuestas de mejora en los planes formativos de estudiantes del Grado de Educación Infantil.

\section{Método}

\section{Participantes}

La población objeto de estudio está compuesta por alumnado de primer, segundo y tercer curso del Grado de Educación Infantil de la Universidad Católica de Murcia $(\mathrm{N}=270)$. No obstante, la muestra está compuesta por 183 alumnos universitarios que participaron en la investigación cumplimentando el cuestionario proporcionado.

Para la selección de los sujetos se ha utilizado un muestreo probabilístico aleatorio simple, de forma que se han adscrito a la muestra todos aquellos sujetos que al azar respondieron a los cuestionarios enviados. La muestra participante se ha calculado según la fórmula para poblaciones con menos de 100.000 sujetos con un nivel de confianza del 95\% y un error de estimación máximo de un 5\%. La siguiente tabla muestra algunas de las características más relevantes de la muestra tales como:

Tabla 1. Datos de la muestra

\begin{tabular}{|c|c|}
\hline Género & $\begin{array}{l}\text { Mujeres: } 62.7 \% \\
\text {. Hombres: } 37.3 \%\end{array}$ \\
\hline Edad & $\begin{array}{l}\cdot \text { 18-21 años: } 54.1 \% \\
\cdot \text { 22-25 años: } 32.1 \% \\
\cdot \text { Más de } 26 \text { años: } 13.8 \%\end{array}$ \\
\hline $\begin{array}{l}\text { Otros estudios } \\
\text { postobligatorios }\end{array}$ & $\begin{array}{l}\text { Si: } 38.3 \% \\
\text { No: } 61.7 \%\end{array}$ \\
\hline $\begin{array}{c}\text { Motivo para acceder a } \\
\text { este Grado }\end{array}$ & $\begin{array}{l}\text { - Motivos vocacionales: } 89.6 \% \\
\text { - Acceder a un puesto de trabajo estable: } 8.2 \% \\
\text { - Adquirir cierto prestigio: } 1.1 \% \\
\text { trayectoria académica: } 1.1 \%\end{array}$ \\
\hline
\end{tabular}




\section{Instrumento}

Para la recogida de datos se ha utilizado la técnica de la encuesta a través del cuestionario AURE "Cuestionario de Autoconcepto y Realización" (Domínguez, 2001). Dicho instrumento, está conformado por 55 ítems agrupados en seis factores, los cuáles recogen la autovaloración del sujeto en contenidos considerados clave para el constructo "Autorrealización": Autoconcepto, Proyectos, Actitud ante la tarea, Amistad y Amor, Preocupación Social y Fuerza del Yo. Se trata de una escala tipo Likert cuyas opciones de respuestas oscilan entre 1 y 5 (siendo $1=$ muy de acuerdo y $5=$ totalmente en desacuerdo).

El cuestionario reúne criterios de fiabilidad, pues el coeficiente obtenido a través del Alfa de Cronbach (.91) y Spearman Brown para mitades iguales (.93) así lo demuestran. Por su parte, el análisis de la estructura factorial aporta una comprensión de los contenidos capaz de reorganizarlos en tres grandes factores tales como:

- Afrontamiento, operatividad y realización en el quehacer: recoge aspectos de planificación, disponibilidad y autocontrol ante la tarea y la norma.

- Autoconcepto y autoestima, que recopila todos los aspectos relacionados con la evaluación de sí mismo y de la autoestima, siendo el factor nuclear en el ajuste con uno mismo.

- Empatía y realización social, que se refiere a la satisfacción en la relación con los otros, la preocupación y la comunicación con los demás.

\section{Resultados}

\section{Análisis descriptivo}

En primer lugar, y teniendo en cuenta la dimensión "Amistad y Amor", los resultados obtenidos demuestran percepciones favorables en el alumnado del Grado de Educación Infantil al cuestionar su conducta hacia los otros y de los otros hacia él. Así pues, los estudiantes afirman alegrarse cuando un conocido obtiene éxito ante cualquier tarea $(M=1.39 ; S D=.779)$, sintiendo especial cariño hacia determinadas personas $(M=1.41 ; S D=.792)$. De igual modo, afirman preocuparse por conocer y tener en cuenta los intereses y gustos de sus amigos $(M=1.48 ; S D=.797)$ así como los problemas e ilusiones de su familiares $(M=1.52 ; S D=.783)$. El alumnado del Grado de Educación Infantil manifiesta colaborar y ayudar a sus amigos ante las dificultades $(M=1.54 ; S D=.812)$, compartiendo su tiempo con otras personas $(\mathrm{M}=1.57 ; \mathrm{SD}=.860)$.

Sin embargo, no le gusta tomarle el pelo a nadie ni burlarse de él $(M=1.76$; $\mathrm{SD}=.925)$, sintiéndose amado y querido por los demás $(M=1.87 ; \mathrm{SD}=.716)$, por lo que suele compartir con éstos sus preocupaciones $(M=2.00 ; S D=.889)$. Considera que es respetado $(M=2.02 ; S D=.774)$, valorado y tenido en cuenta por los demás $(M=2.10 ; S D=.787)$, por lo que muchas veces recibe favores y ayuda de éstos $(\mathrm{M}=2.12 ; \mathrm{SD}=.798)$.

Para la dimensión "Proyectos", los resultados muestran percepciones favorables por parte del alumnado encuestado. Así pues, suele pasarlo bien imaginando futu- 


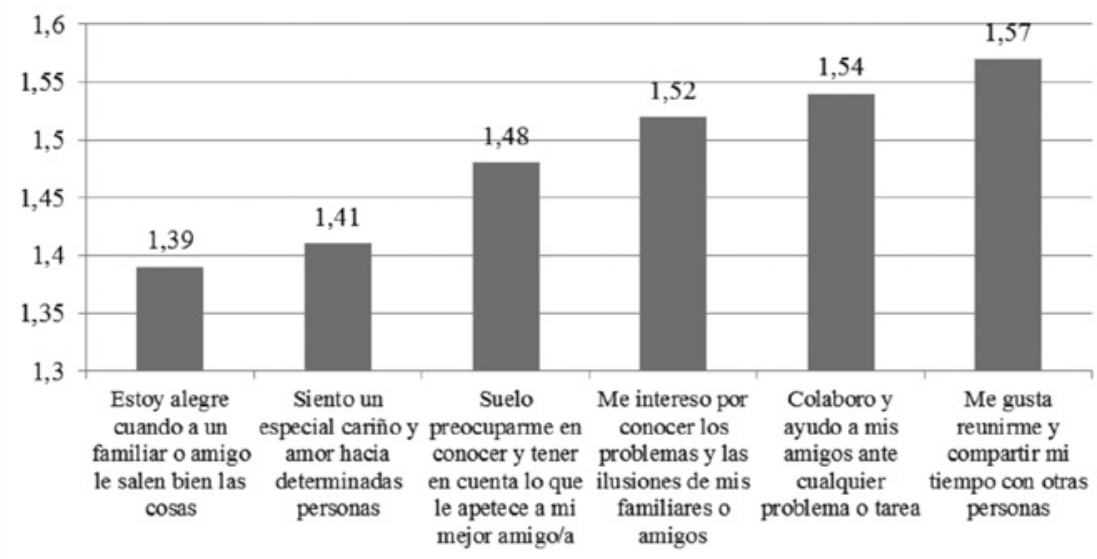

Figura 1. Análisis descriptivo para la dimensión "Amistad y Amor" (I)

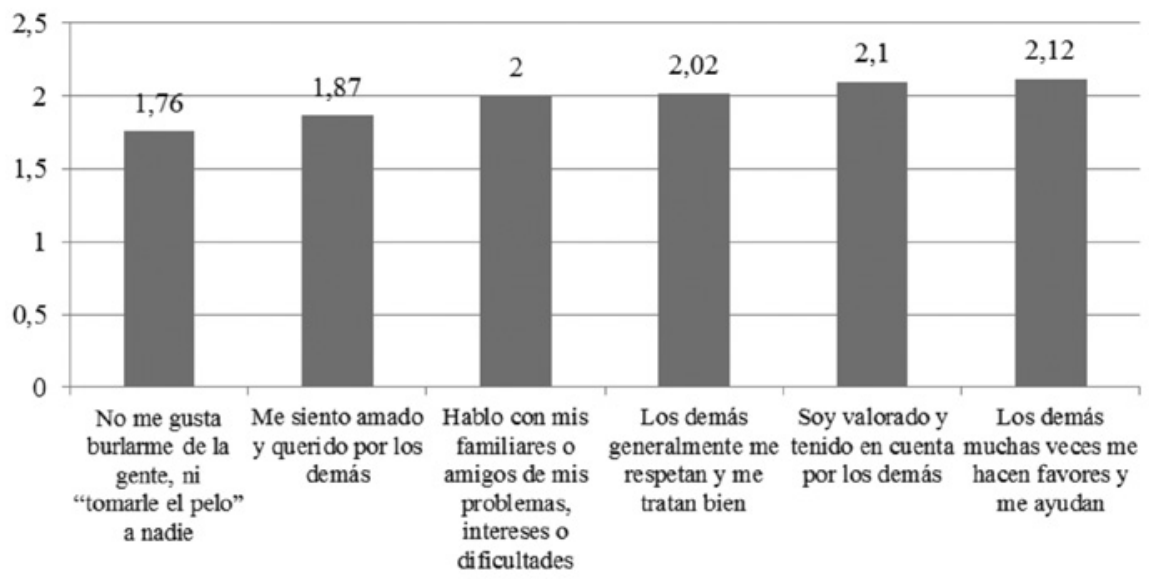

Figura 2. Análisis descriptivo para la dimensión "Amistad y Amor" (II)

ros proyectos $(M=1.86 ; S D=.907)$, haciéndolo con frecuencia $(M=2.04 ; S D=.910)$. Además, le resulta interesante y le entretiene todo aquello que ocurre a su alrededor $(M=2.19 ; \mathrm{SD}=.749)$. Afirma pasarlo bien realizando determinados proyectos $(M=2.20 ; S D=.824)$, siendo éstos realistas y fáciles de ejecutar $(M=2.34: S D=.771)$. No obstante, disfruta más actuando y desarrollándolos que pensando sobre ellos $(M=2.39 ; S D=.837)$, por lo que generalmente lo lleva a la práctica $(M=2.41$; $\mathrm{SD}=.802)$.

Centrados en la dimensión "Actitud ante la tarea", el alumnado del Grado de Educación Infantil participante en la investigación afirma revisar el trabajo realizado para detectar posibles errores $(M=1.80 ; S D=.761)$, siendo persistente y esforzándose 


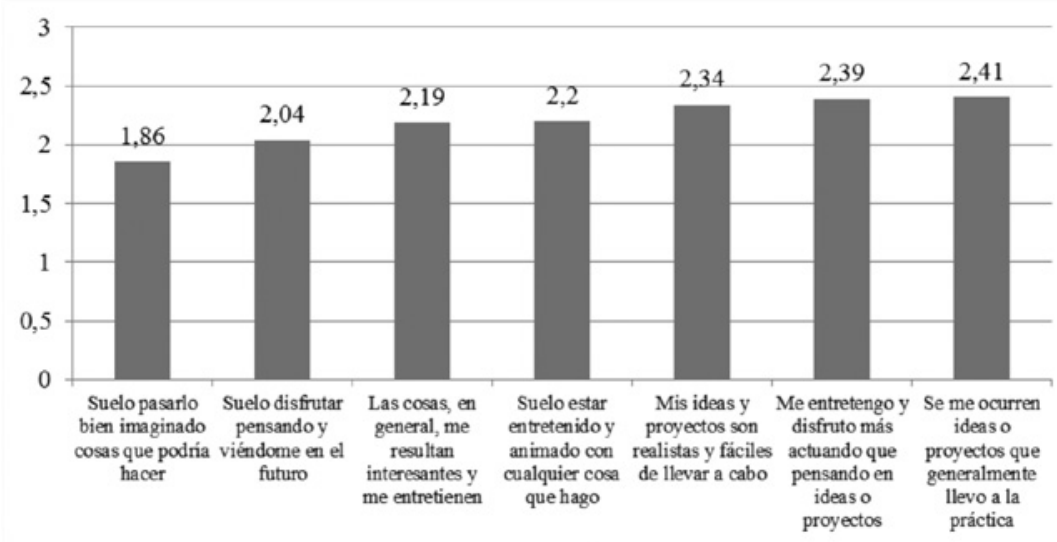

Figura 3. Análisis descriptivo para la dimensión "Proyectos"

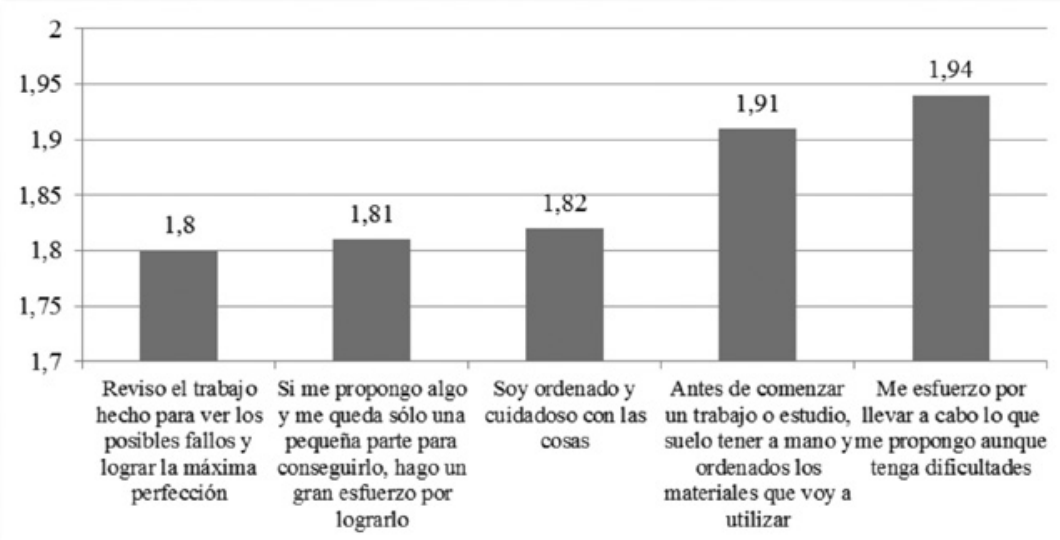

Figura 4. Análisis descriptivo para la dimensión "Actitud ante la tarea" (I)

por finalizar las tareas $(M=1.81 ; S D=.840)$. Se considera una persona ordenada y cuidadosa con las cosas $(M=1.82 ; \mathrm{SD}=.997)$, por lo que antes de comenzar a trabajar, prepara todo lo necesario $(M=1.91 ; \mathrm{SD}=.906)$ y planifica lo que va a hacer y cómo lo va a desarrollar $(M=1.96 ; \mathrm{SD}=.848)$.

Manifiesta esforzarse ante las dificultades $(M=1.94 ; S D=.820)$, teniendo buena disponibilidad e interés por realizar cualquier trabajo $(M=2.01 ; S D=.880)$, consultando y aclarando lo que no entiende y revisando los errores $(M=2.01 ; S D=.788)$. El alumnado afirma tener confianza en sus posibilidades $(M=2.05 ; \mathrm{SD}=.834)$, prefiriendo desarrollar tareas que supongan nuevos retos ( $M=2.39 ; \mathrm{SD}=.896)$.

Para la dimensión "Autoconcepto", los resultados obtenidos muestran percepciones favorables para casi la totalidad de los ítems. Así pues, el alumnado del Grado de Educación Infantil encuestado se considera una persona trabajadora $(M=1.58$; 
$\mathrm{SD}=.780)$, amable con los demás $(M=1.69 ; \mathrm{SD}=.801)$, disciplinado $(M=1.71$; $\mathrm{SD}=.804)$, obediente $(M=1.79 ; \mathrm{SD}=.832)$, alegre y divertido $(M=1.80 ; S D=.824)$. Así pues, se siente satisfecho consigo mismo $(M=1.81 ; S D=.735)$, queriendo ser tal y como es $(\mathrm{M}=1.87 ; \mathrm{SD}=.873)$.

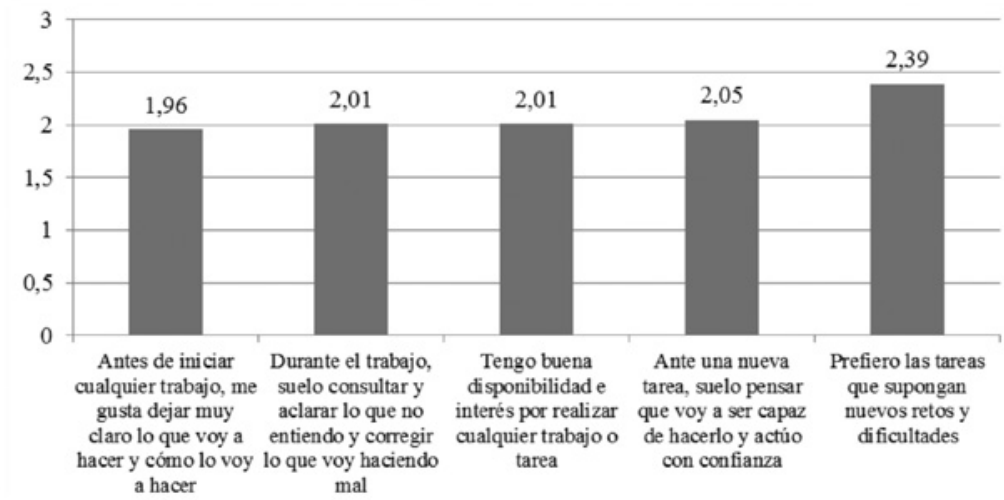

Figura 5. Análisis descriptivo para la dimensión "Actitud ante la tarea" (II)

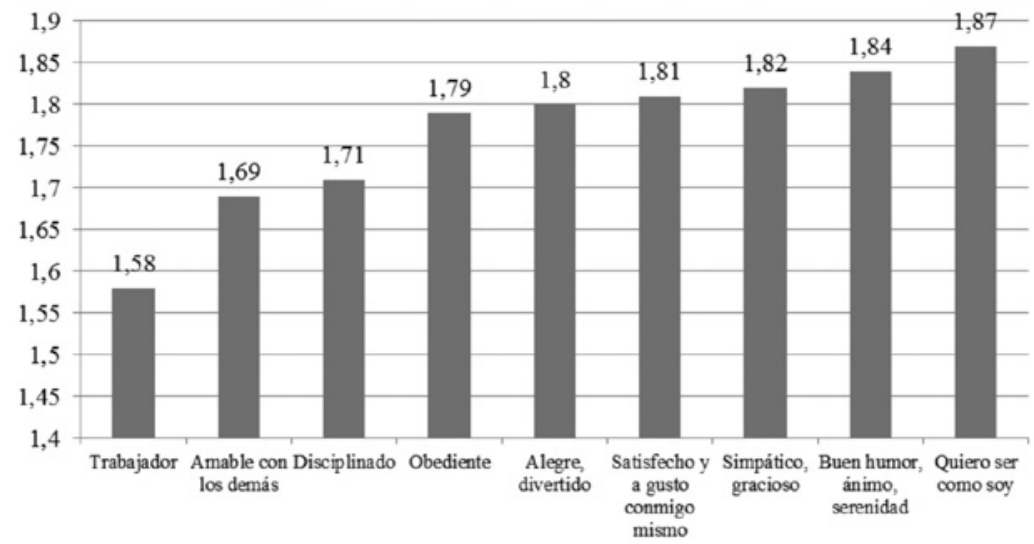

Figura 6. Análisis descriptivo para la dimensión "Autoconcepto" (I)

Se define como una persona simpática $(M=1.82 ; \mathrm{SD}=.776)$, con buen sentido del humor $(M=1.84 ; S D=, 802)$, saludable $(M=1.99 ; S D=.961)$, con muchas cualidades $(M=2.04 ; S D=753)$; es valiente $(M=2.22 ; S D=914)$, inteligente $(M=2.27 ; S D=.716)$, guapa $(M=2.42 ; S D=.840)$ y decidida $(M=2.47 ; S D=1.063)$. No obstante, muestra su indiferencia al cuestionar su suerte o fortuna $(M=2.83 ; S D=.793)$ y valorar su fortaleza a nivel físico $(M=2.96 ; S D=.928)$. 


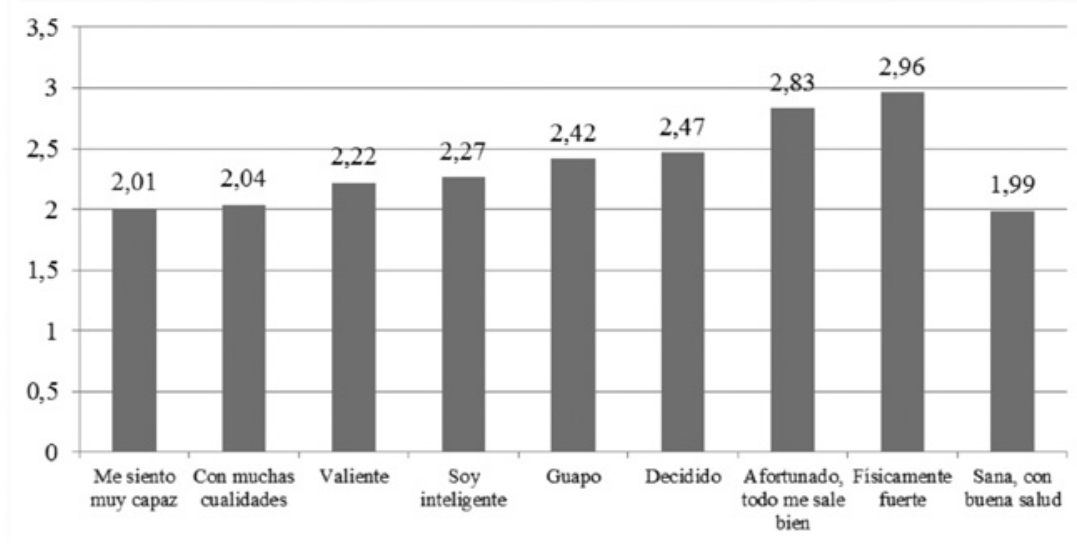

Figura 7. Análisis descriptivo para la dimensión "Autoconcepto" (II)

Para la dimensión "Preocupación Social", los resultados obtenidos demuestran cómo el alumnado considera que puede ayudar a solucionar determinados problemas de los demás ( $M=2.02 ; \mathrm{SD}=.756)$, pues es algo que le preocupa $(M=2.07 ; \mathrm{SD}=.809)$ y suele hablarlo con las personas de su entorno $(M=2.14 ; S D=.794)$. No obstante, se muestra algo indiferente al cuestionar la puesta en práctica de determinadas actividades de ayuda para solucionar los problemas que le rodean $(M=2.57 ; \mathrm{SD}=.1 .599)$.

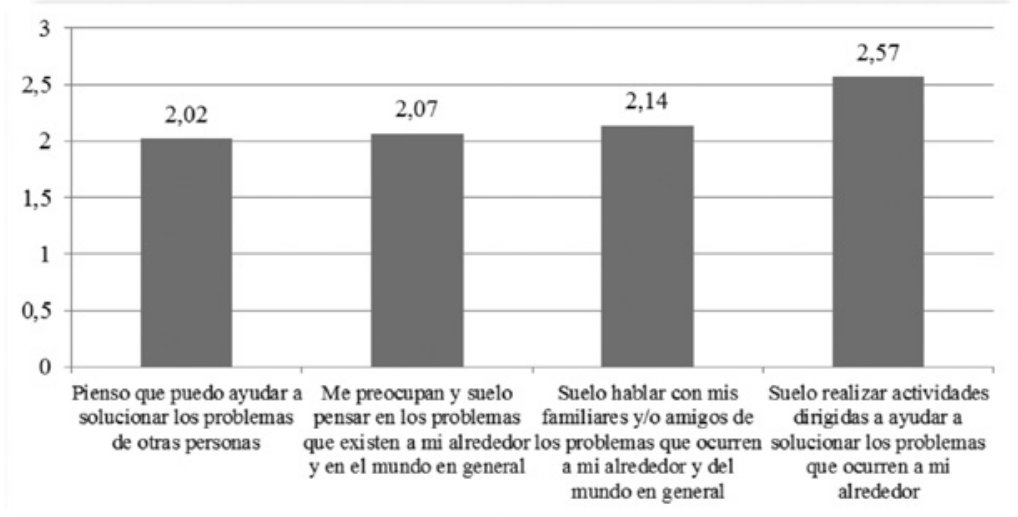

Figura 8. Análisis descriptivo para la dimensión "Preocupación Social"

Finalmente, y vinculada con la dimensión "Fuerza del Yo", los resultados demuestran la constancia y persistencia del alumnado encuestado hacia la tarea $(M=1.97 ; S D=.894)$, tolerancia y paciencia ante las dificultades $(M=2.05 ; S D=.927)$. Asimismo, manifiesta desarrollar habilidades de autocontrol ante los impedimentos de otras personas $(M=2.30 ; S D=.955)$ y búsqueda de alternativas ante los fracasos $(\mathrm{M}=2.40 ; \mathrm{SD}=.877)$. 


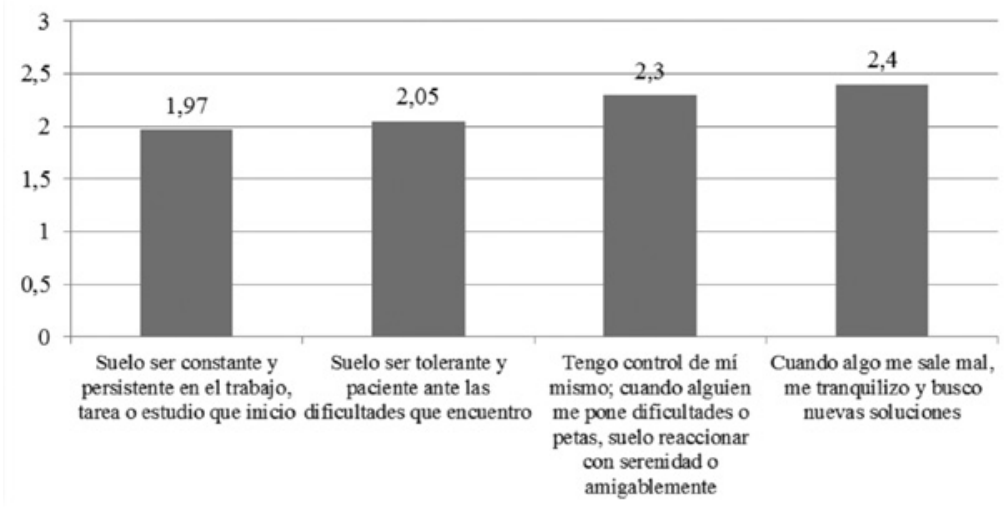

Figura 9. Análisis descriptivo para la dimensión "Fuerza del Yo"

Una vez realizado el análisis descriptivo de aquellos ítems cuyos resultados han sido más significativos, se analiza la relación entre los factores que agrupan los ítems del cuestionario mediante la correlación r de Pearson (tabla 2) siendo éstas: Amistad y Amor, Actitud ante la tarea, Autoconcepto, Fuerza del Yo, Proyectos y Preocupación Social. Dicho coeficiente de correlación es el mejor y más utilizado para estudiar el grado de relación lineal existente entre dos variables cuantitativas, tomando valores entre -1 y 1 . Así pues, se ha rechazado la hipótesis nula de independencia cuando el nivel crítico es menor que el nivel de significación (.05).

Los resultados demuestran cómo las mayores correlaciones se sitúan entre la dimensión "Autoconcepto" y "Amistad y Amor" (.781), "Actitud ante la tarea" (.719), "Proyectos" (.670) y "Fuerza del Yo" (.635), obteniendo valores algo más bajos para la dimensión "Preocupación Social" (.396).

Ello demuestra la relación existente entre el autoconcepto de los estudiantes del Grado de Educación Infantil, es decir, nivel de satisfacción personal, valoración física, capacidad intelectual, estado de ánimo, interacción social, enfrentamiento con la realidad, adaptación normativa y la conducta del estudiante hacia los otros (sociabilidad y comunicación, respeto, colaboración e identificación) y de los otros hacia él (afecto, valoración positiva, respeto, colaboración y ayuda). Además, muestra correlaciones entre su autoconcepto y la actitud ante la tarea, valorando tanto la afectividad (disponibilidad ante la tarea, motivación y evaluación de logro) como la efectividad (planificación, precisión de la acción y evaluación del trabajo) así como el potencial de disfrute y la adecuación de los proyectos realizados. Finalmente, se aprecian correlaciones entre la valoración personal de los estudiantes y su nivel de austeridad, disponibilidad al esfuerzo, persistencia, búsqueda de alternativas, tolerancia social y autonomía.

Para la dimensión "Proyectos", se aprecian correlaciones favorables entre ésta y la dimensión "Actitud ante la tarea" (.618), "Amistad y Amor" (.568), "Fuerza del Yo" (.555) y "Preocupación Social" (.521), centrada en el análisis del nivel de preocupación y acción de los estudiantes. Por su parte, y para la dimensión "Actitud ante la tarea", se comprueba reciprocidad para la dimensión "Amistad y Amor" (.662) y 
"Fuerza del Yo" (.654), no ocurriendo lo mismo para la dimensión "Preocupación Social" (.458). La dimensión "Amistad y Amor" muestra correlaciones significativas con la dimensión "Fuerza del Yo" (.572) y "Preocupación Social" (.532); además, ésta última correlaciona con la dimensión "Fuerza del Yo" (.420).

Tabla 2. Correlaciones entre los factores de la escala

\begin{tabular}{|c|c|c|c|c|c|c|}
\hline \multicolumn{7}{|c|}{$r^{*}$} \\
\hline & Autonconcepto & Proyectos & $\begin{array}{c}\text { Actitud } \\
\text { ante la } \\
\text { tarea }\end{array}$ & $\begin{array}{l}\text { Amistad } \\
\text { y Amor }\end{array}$ & $\begin{array}{l}\text { Preocupación } \\
\text { Social }\end{array}$ & $\begin{array}{c}\text { Fuerza } \\
\text { del Yo }\end{array}$ \\
\hline Autoconcepto & & .670 & .719 & .781 & .396 & .635 \\
\hline Proyectos & .670 & & .618 & .568 & .521 & .555 \\
\hline Actitud ante la tarea & .719 & .618 & & .662 & .458 & .654 \\
\hline Amistad y Amor & .781 & .568 & .662 & & .532 & .572 \\
\hline Preocupación Social & .396 & .521 & .458 & .532 & & .420 \\
\hline Fuerza del Yo & .635 & .555 & .654 & .572 & .420 & \\
\hline
\end{tabular}

* La correlación es significativa a nivel 0.05 (bilateral).

\section{Discusión y CONCLUSIONES}

Este trabajo ha permitido analizar las percepciones sobre valores asociados al autoconcepto y la realización personal en estudiantes del Grado de Educación Infantil. Dicha investigación puede servir de interés para el análisis de este campo de estudio y, a partir de él, proponer el desarrollo de experiencias de aprendizaje basadas en nuevas técnicas de atención consciente "mindfulness" en los planes de estudios propuestos para la formación inicial del profesorado. Dichas técnicas provocan alegría y felicidad en la persona que lo practica de manera regular, además de otras emociones positivas, aumento de resistencia física y mental y relaciones sociales más satisfactorias.

Los resultados obtenidos muestran las percepciones favorables de los estudiantes del Grado de Educación Infantil hacia los valores de autoconcepto y realización personal. Ello va a tener una repercusión positiva en su futuro desarrollo profesional, pues dispone de unas adecuadas habilidades y competencias vinculadas a la imagen que el estudiante tiene de sí mismo y su relación con el entorno y los demás. Además, y tal y como afirma Pool y Sewell (2007), el desarrollo de la competencia emocional permite un aprovechamiento del potencial de aprendizaje en los universitarios.

Así pues, el proceso formativo del docente en el ámbito universitario requiere no sólo de contenidos teóricos que le habiliten para el dominio de conceptos, hechos y principios, sino que es necesario la inclusión de competencias emocionales (López y Goñi, 2012), centradas en la conciencia y capacidad de gestión y control de emociones y sentimientos, motivación y relaciones interpersonales (Soriano y Osorio, 2008).

Los estudiantes encuestados afirman sentirse satisfechos en su relación con los otros, comunicación con los demás y preocupación por los problemas de su alrede- 
dor aunque cuestionan la puesta en práctica de actividades de ayuda para darle solución. Ello revela cómo los alumnos muestran una inquietud prosocial colaborativa, aunque denota falta de disposición para ejecutar determinadas medidas que pretendan solucionar dichos problemas. Disponen de habilidades para la planificación, disponibilidad y autocontrol ante la tarea y la norma. Además, poseen una evaluación positiva y ajustada de sí mismo, mostrándose algo indiferente al cuestionar su fuerza física y suerte en la vida.

Además, los resultados demuestran cómo las mayores correlaciones se sitúan entre las dimensiones "Autoconcepto", "Amistad y Amor", "Actitud ante la tarea", "Proyectos" y "Fuerza del Yo". Ello pone de manifiesto cómo la imagen que el estudiante tiene de sí mismo influye en sus relaciones con el grupo de iguales, su predisposición ante la tarea y la norma, ejecución de proyectos y autopercepción sobre su nivel de austeridad, disponibilidad al esfuerzo, persistencia, búsqueda de alternativas, tolerancia social y autonomía en el contexto en que se desarrolla. Ello concuerda con lo expuesto por Bisquerra (2007) quién muestra cómo la adquisición de competencias emocionales en el profesorado beneficia las relaciones que éste establece con el alumnado y resto de comunidad educativa; además, el docente competente emocionalmente es capaz de establecer una serie de estrategias activas para enfrentarse a situaciones estresantes dentro del entorno académico (Mearns y Cain, 2005).

Estos resultados entran en contradicción con los aportados por Hué (2008) quién alude al malestar y desánimo profesional del docente y su incidencia sobre su nivel de autoconcepto, inseguridad y falta de autoestima. Igualmente, Peñalva, López y Landa (2013) han subrayado el déficits en cuanto al desarrollo de habilidades personales e interpersonales en estudiantes de Magisterio, lo que incide de manera negativa en la integración laboral exitosa de estos estudiantes (Pertegal, Castejón y Martínez, 2009). Así pues, los estudiantes con actitudes negativas hacia sí mismos se imponen mayor grado de limitaciones para sus logros (Naranjo, 2007), pues el autoconcepto académico y social negativo están asociados a un rendimiento académico bajo (De la Orden y González, 2005).

No obstante, y como posibles limitaciones del estudio, se destaca cómo el uso exclusivo del cuestionario como instrumento de recogida de datos puede causar problemas de sinceridad y deseabilidad social entre los encuestados. Además, la puesta en práctica de tal investigación en el contexto de la Universidad Católica de Murcia puede perjudicar la generalización de datos para estudiantes del Grado de Maestro de otras universidades.

A partir de dicho análisis, es posible la puesta en práctica de experiencias de aprendizaje basadas en nuevas técnicas de atención consciente "mindfulness". Estos deben integrarse en los planes formativos del profesorado de Educación Infantil para favorecer el desarrollo de los niveles de autoaceptación y realización personal en los estudiantes. Se debe producir, pues, una mejora de las percepciones y valoraciones de los estudiantes del Grado de Maestro acerca de las habilidades emocionales de que disponen para así responder a determinados problemas que adolecen al sistema educativo y social en la actualidad.

Tales experiencias deben Ilevarse a cabo a partir del diseño de un instrumento de recogida de datos sobre atención plena "mindfulness" que permita valorar las 
percepciones del alumnado tras el desarrollo de tales prácticas. La propuesta llevada a cabo en el aula universitaria con estudiantes del Grado de Educación Infantil debe tener en cuenta una serie de principios, tal y como define Kaiser (2010), siendo éstos:

- Definir con claridad y justificar por qué se está enseñando mindfulness en el aula.

- Su enseñanza debe ser laica, dejando a un lado cualquier implicación de carácter religioso.

- Utilizar un lenguaje simple y caro que comunique e instruya las actividades de la forma más clara posible.

- Pretende llevar al juego y la diversión a partir de actividades como el canto, baile, risa o juegos.

- Integrar mindfulness en las actividades cotidianas.

- Colaboración, pues se tratar de apartar nuestras diferencias y pensamientos personales para trabajar juntos por el beneficio de todos (estudiantes, profesores y familias).

- Desarrollar una estrategia global de investigación y desarrollo de programas de atención plena dignos de ser aplicados en lugares públicos.

Asimismo, resulta interesante tener en consideración determinadas variables como pueda ser el rendimiento académico y/o motivación académica y personal del alumnado. Se tratan de aspectos de vital importancia para el análisis del alumnado universitario, que pueden resultar beneficiados tras la práctica de tales experiencias en el aula universitaria.

Por ello, y para futuras investigaciones, se pretende ampliar la muestra objeto de estudio con alumnado del Grado de Educación Infantil de otras universidades (nacionales e internacionales), así como por establecer relaciones entre los niveles de autorrealización y etapas educativas (Educación Infantil, Primaria, Secundaria, Universidad, etc.). Además, la formación de grupos de discusión entre alumnado permite el análisis cualitativo de la información obtenida acerca de las percepciones en los estudiantes sobre autoconcepto y realización personal.

Tal y como manifiestan Ramírez y De la Herrán (2012), para favorecer el desarrollo profesional del docente es necesario trabajar desde la raíz, es decir, desde la madurez personal, siendo consciente de lo que ello condiciona para la enseñanza y el propio desarrollo profesional del docente. Así pues, es deseable que la educación conlleve la autorrealización personal, abarcando desde la protección de los sentimientos, emociones y autoestima en el alumnado, hasta la ampliación de sus oportunidades de elegir, reconocimiento de su singularidad, identidad colectiva, personalidad individual y de su particularidad cultural (Fernández y Terrén, 2008).

\section{REFERENCIAS BIBLIOGRÁFICAS}

Bisquerra, R. (2000). Educación emocional y bienestar. Barcelona: Praxis.

Bisquerra, R. (2007). La educación emocional en la formación del profesorado. Revista Interuniversitaria de Formación del Profesorado, 54, 95-114. 
Bueno, C., Teruel, P. y Valero, A. (2005). La inteligencia emocional en alumnos de Magisterio: la percepción y comprensión de los sentimientos y las emociones. Revista Interuniversitaria de Formación del Profesorado, 19(3), 169-194.

Burnett, R. (2011). Mindfulness in schools, learning lessons from the adults-secular and Buddhist. Buddhist Studies Review, 28(1), 79-120. Recuperado de: http:// www.mindfulnet.org/Mindfulness_in_Schools_Burnett_2009.pdf.

Cassullo, G. L. y García, L. (2015). Estudio de las Competencias Socio Emocionales y su Relación con el Afrontamiento en Futuros Profesores de Nivel Medio. Revista Electrónica Interuniversitaria de Formación del Profesorado, 18(1), 213-228.

De la Orden, A. y González, C. (2005). Variables que discriminan entre alumnos de bajo y medio-alto rendimiento académico. Revista de Investigación Educativa, 23, 573-599. Doi:10-4438/1988-592X-RE-2011-362-162.

Domínguez, M. C. y García, P. (2012). Tratamiento didáctico de las competencias básicas. Madrid: Universitas.

Domínguez, R. (2001). Intervención para la realización personal y social: validación de un instrumento de diagnóstico y evaluación del cambio. Tesis doctoral. Universidad de La Laguna.

Extremera, N. y Fernández-Berrocal, P. (2004). El papel de la inteligencia emocional en el alumnado: evidencias empíricas. Revista Electrónica de Investigación Educativa, 6(2), 1-17. Recuperado de: http://www.redalyc.org/pdf/155/15506205. pdf.

Fernández, M. R., Palomero, J. E. y Teruel, M. P. (2009). El desarrollo socioafectivo en la formación inicial de los maestros. Revista Interuniversitaria de Formación del Profesorado, 12(1), 33-50.

Fernández, M. y Terrén, E. (2008). De inmigrantes a minorías: temas y problemas de la multiculturalidad. Revista de Educación, 345, 15-21.

Franco, C., De la Fuente, M. y Salvador, M. (2011). Impacto de un programa de entrenamiento en conciencia plena (mindfulness) en las medidas de crecimiento y la autorrealización personal. Psicothema, 23, 58-65.

Hué, C. (2008). Bienestar docente y pensamiento emocional. Madrid: Wolters Kluwer.

Kaiser, S. A. (2010). Mindful revolution in education. Recuperado de: http:// www.huffingtonpost.com/susan-kaiser-greenland/a-mindful-revolutionine_b_680435.html.

Langer, A., Ulloa, V., Cangas, A., Rojas, G. y Krause, M. (2015). Mindfulness-based interventions in secondary education: a qualitative systematic review. Studies in Psychology, 36(3), 533-570.

López, I. y Goñi, J. M. (2012). La competencia emocional en los currículos de formación inicial de los docentes. Un estudio comparativo. Revista de Educación, 357, 205-206.

Martínez, J. A. (2015). Nuevos roles del profesor y del estudiante universitario en el contexto del Espacio Europeo de Educación Superior. Aplicación al área de organización de empresas. Cuadernos de Educación y Desarrollo, 55. Recuperado de: http://atlante.eumed.net/wp-content/uploads/competencias1.pdf. 
Mayer, J. D., Salovey, P. y Caruso, D. (2000). Models of emotional intelligence. En R. J. Sternberg (eds.). Handbook of intelligence (pp. 396-420). New York: Cambridge University Press.

Mearns, J. y Cain, J. E. (2003). Relationships between teachers'occupational stress and their burnout and distress:roles of coping and negative mood regulation expectancies, Anxiety, Stress and Coping, 16, 71-82.

Medina, A., Domínguez, M. C. y Sánchez, C. (2013). Evaluación de las competencias de los estudiantes: modelos y técnicas para la valoración. Revista de Investigación en Educación, 31(1), 239-256. DOI: http://doi.org/10.6018/rie.31.1.157601.

Meiklejohn, J., Phillips, C., Freedman, M. L., Griffin, M. L., Biegel, G., Roach, A., Frank, J. Burke, Ch., Pinger, L., Soloway, G., Isberg, R., Sibinga, E., Grossman, L. y Saltzman, A. (2012). Integrating mindfulness training into K-12 education: Fostering the resilience of teachers and students. Mindfulness, 3(4), 291-207. DOI: http://doi.org/10.1007/s12671-012-0094-5.

Mieles, M., Henriquez, I. y Sánchez, L. (2009). Identidad personal y profesional de los docentes de preescolar en el distrito de Santa Marta. Educación y Educadores, 12(1), 43-59.

Naranjo, M. P. (2007). Autoestima: un factor relevante en la vida de la persona y tema esencial del proceso educativo. Actualidades Investigativas en Educación, 7, 1-27.

Palomares, A. y Garrote, D. (2009). Un nuevo modelo docente por y para el alumnado. Ensayos: Revista de la Facultad de Educación de Albacete, 24, 25-34.

Palomera, R., Fernández-Berrocal, P. y Brackett, M. A. (2008). La inteligencia emocional como una competencia básica en la formación inicial de los docentes: algunas evidencias. Revista Electrónica de Investigación Psicoeducativa, 15(6), 437-454.

Pena, M. y Extremera, N. (2012). Inteligencia emocional percibida en profesorado de Primaria y su relación con los niveles de burnout e ilusión por el trabajo (engagement). Revista de educación, 359, 604-627.

Peñalva, A., López, J. J. y Landa, N. (2013). Competencias emocionales del alumnado de Magisterio. Posibles implicaciones profesionales. Revista de Educación, 362, 690-712.

Pertegal, M. L., Castejón, J. L. y Martínez, M. A. (2009). Competencias socioemocionales en el desarrollo profesional del Maestro. Educación XXI, 14(2), 237-260. DOI: http://doi.org/10.5944/educxx1.14.2.253.

Pool, L. y Sewell, P. (2007). The key to employability: developing a practical model of graduate employability. Education \& Training, 49(4), 277-289.

Poulou, M. (2005). The Prevention of Emotional and Behavioural Difficulties in Schools: Teachers' suggestions. Educational Psychology in Pratice, 21, 37-52. DOI: http://doi.org/10.1080/02667360500035181.

Ramírez, M. S. y De la Herrán, A. (2012). La madurez personal en el desarrollo profesional del docente. REICE: Revista Electrónica Iberoamericana sobre Calidad, Eficacia y Cambio en Educación, 10(3), 25-44.

Salami, S. O. (2010). Emotional intelligence, self-efficacy, psychological well-being and students' attitudes: implications for quality implications. European Journal of Educational Studies, 2(3), 247-257. 
Shapiro, S. L., Brown, K. W. y Astin, J. A. (2008). Toward the integration of meditation into higuer education. A review of research. Recuperado de: http://www.colorado. edu/ftep/events/eventdocs/documents/ShapiroResearchReport.pdf.

Sternberg, R. (1997). Inteligencia exitosa: cómo una inteligencia práctica y creativa determina el éxito en la vida. Barcelona: Paidós.

Soriano, E. y Osorio, M. M. (2008). Competencias socioemocionales del alumnado "autóctono" e inmigrante de Educación Secundaria. Bordón, 60(1), 129-148.

Thera, H. (2008). El cultivo de la atención plena: La práctica de la meditación vipassana. México: Pax.

Zabalza, M. A. (2006). La Universidad y la docencia en el mundo de hoy. Bogotá: Pontificia Universidad Javeriana. 